\title{
An Evaluation Method of Event Information for Efficient Data Fusion
}

\author{
Donghyok Suh and Juhye Yook* \\ Department of Multimedia Communication, Far East University, \\ *Professor, Department of Rehabilitation Technology, Korea Nazarene University \\ hanhwaco@naver.com, jhyook@kornu.ac.kr
}

\begin{abstract}
Multi-sensors data fusion takes center stage in wireless sensor network system for acquiring more precise information. The event information detected and reported by sensors is often not reliable to infer contexts. The study presents that the data are evaluated before the data fusion on all event information acquired is occurred in the aggregate. The evaluation of event information is to estimate if context inference is possible and to reduce the processes of unnecessary multi-sensors data fusion. The study shows the frequency of event occurrences and the average variation rates of the data reported by the sensors for evaluating event information as the evaluation criteria. As a result of evaluating event information based on the suggested evaluation criteria and processing data fusion selectively, unnecessary data fusion processes were reduced, the data processes for context inference were decreased, and eventually the efficiency of context inference was improved.
\end{abstract}

Keywords: Context Awareness, Context Inference, Data fusion

\section{Introduction}

The assured reliability of signals detected by sensors is essential in the studies on the context awareness based on the signals transmitted from a large number of wirelessly networked multi-sensors. Sensors detect the information on targets and surrounding environments and report these to the host. Then the event information detected by sensors is filtered at first and determined if it is worthwhile to report based on certain criteria. The sensor motes transmit the event information detected to the host if the event is determined worthwhile to report. Not all of the event information reported to the host is appropriate for the next data processes even if the event information is passed the first filtering. Data processes in wireless sensor network after data filtering are data aggregation and data fusion. Data aggregation is to eliminate overlaps while data fusion is to aware context information more precisely. Data fusion for only validated event information would be beneficial in the resource use of the system as data aggregation eliminates overlapped data to use network and host resources efficiently. Evaluating if the reported event information has sufficient grounds to reach to context inference and conducting additional improvement of insufficient event information would save the resources of the system and infer context information better with multi-sensors data fusion based on improved event information.

* Corresponding Author 
Moreover, event information should be validated because the values reported by sensors are often inaccurate and in error. The problems might occur in sensors' detection phases and in errors and decreases for communication network difficulties while reporting sensing results. For these reasons, it is inevitable that the reliability of detected signals from sensors is damaged somewhat. So, the reliability of the observed values from sensors should be improved to increase the quality of context awareness based on sensor signals.

The study presents a method to evaluate the reliability of signals for improving the performance of context awareness. Changing circumstances as time changes should be detected for correct context awareness. Sensors report sensing results in each preset interval, and the signals are evaluated before conducting multi-sensors data fusion in each internal. The signals with low reliability are evaluated in the next intervals repeatedly to assure the increase of their reliability and to use for context awareness because multi-sensors data fusion for context awareness is processed continually. The study also presents how to evaluate sensing data with what criteria before conducting multi-sensors data fusion. Data fusion is undertaken with the signals that passed reliability criteria, and the signals with low level of reliability are reevaluated in the next intervals. These procedures improve the instability and inaccuracy of the signal values.

The study is consisted of analyzing related research in Chapter 2, evaluating event information for efficient data fusion in Chapter 3, describing the experiments and evaluation in Chapter 4, and discussing conclusion in Chapter 5.

\section{Related Research}

Various researches on multi-sensors use for context awareness have been undertaken. Ontology is the mostly used method for context awareness. Diverse context awareness frameworks generalized by context awareness service are suggested and the examples are as follows.

SOCAM (Service-Oriented Context-Aware Middleware) provides with mobile service using context information translator [1]. The context information translator obtains context information from diffuse context information providers, appropriately processes, and then provides with services. Mobile context awareness service determines its action using different context information. SOCAM framework is the study on context process based on ontology and expression of formularized context. This framework has difficulties in securing the performance of ontology inference engine and scalability.

The framework of CoBrA (Context Broker Architecture) provides with context awareness service based on agents in smart space [2]. Context information broker is in the center of architecture to help acquire context information from different agents, to manage and maintain context information model, and to assist knowledge sharing among agents. The context information broker has roles of knowledge base, inference engine, acquisition module, and security management module. The study on the CoBrA framework is only about the expression and process of obtained sensing data so far and in need of further study on context information acquisition and transmission. The framework is also difficult to have scalability.

Context managing framework has 4 main functions of context information manager, resource server, context awareness service, and application service and 2 subfunctions of change detection and security [3]. This is also in the lack of scalability even if it provides with context information interpretation using inference function and has abundant resources and computing capability. 
GAIA was developed as middleware structure for user-centered environment and defined user activity domains as active spaces. Services are exposed on the outside for the purposes of questioning and using the status of resources in physical spaces or devices and also approaching to and using context information [4]. This composes 4 building blocks of kernel, application framework, QoS service, and application service. GAIA framework can provide with context awareness service through continually monitoring users and environment changes. However, the problem lies on the lack of supporting the actual application for users' real situations.

\section{Evaluating Event Information for Efficient Data Fusion}

\section{The Context awareness}

Existing methods for context awareness using multi-sensors were based on premodeled data set and framework. The important task was to secure various and abundant prior information on real situations targeted by context awareness system. The real world, however, is very diverse and often out of range from pre-modeling, in which the situation may be critical. Therefore, the way to complement is needed for the cases beyond the range of prior information [5].

\section{Time changes and context awareness}

Time changes are taken into consideration for context awareness. It might be easy to recognize static circumstances but would be less important to use with time passing by. The real world changes and is variable as time passes by, and the time could be a factor to compose of situation [6]. Sensing in preset and consistent intervals would be undertaken instead of continuous sensing to avoid excessive consuming sensor resources. It is reported to a host after determined if the sensing values in each interval are corresponding to events and worth to report. It is inefficient to process data without verifying the sensing values collected and reported in each interval or having criteria for fusion because it would consume resources in sensor network system and not obtain reliable context information [7].

\section{Enhancing the reliability of sensor signals}

Awareness activity is carried out in various circumstances because sensors are placed in various places. This implies that the detecting activities of sensors have possibilities of errors and difficulties. The problems might be also in the process of transmitting when the signals detected by sensors are reported to a host through wireless network. Wireless network may have problems in difficulties of communication lines, noises in communicating, and decreases in communication conditions. In addition, sensors for themselves might react differently for certain situations. For example, events occur in $\mathrm{CO}_{2}$ sensors for early phases, but the humidity sensors might not be sensitive in the case of a forest fire. Temperature sensors and humidity sensors would activate events more frequently as the forest fire approaches. So, the signals by sensors from a certain situation need precise evaluation.

The signals detected by sensors are input through relay nodes and sink nodes as in Figure 1. Sensing data are processed for fusion before used as service in a host. The Figure 1 shows sensing and reporting from multi-sensors. 


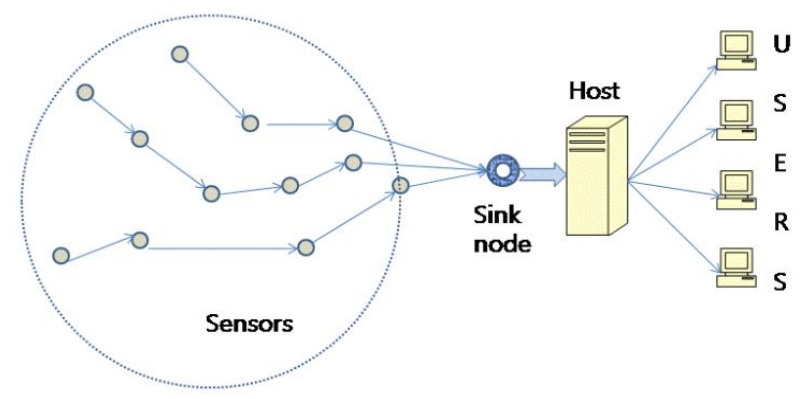

Figure 1. Sensing and reporting from multi-sensors

\subsection{Evaluating event information}

The study shows that it is valid to evaluate sensing data before processing fusion. Then, the issues here are when to evaluate with what criteria. The evaluation takes place before data fusion of signals from sensors to determine what values are appropriate and reliable for data fusion. It is appropriate to evaluate event information in the middleware of the sink nodes after overlapped data are eliminated by data aggregation. Refined event information data are processed for data fusion after overlapped data are eliminated from the event data through the middleware nodes in the sensor nodes.

\subsection{Purposes to evaluate event information}

The purposes to evaluate event information in the study are as the following. Firstly, unnecessary fusion processes are reduced by identifying inappropriate information with errors and imprecise event information. As a result, the data processes become efficient, and the event information for data fusion is a material for determination with high quality. Therefore, the inference functions for context awareness are at a higher level by data fusion with proper event information to infer.

Secondly, the functions of context inference are strengthened by using refined and supplemented event information with evaluation. This is accomplished after performing the first purpose. The Figure 2 displays how to evaluate signal values from sensors.
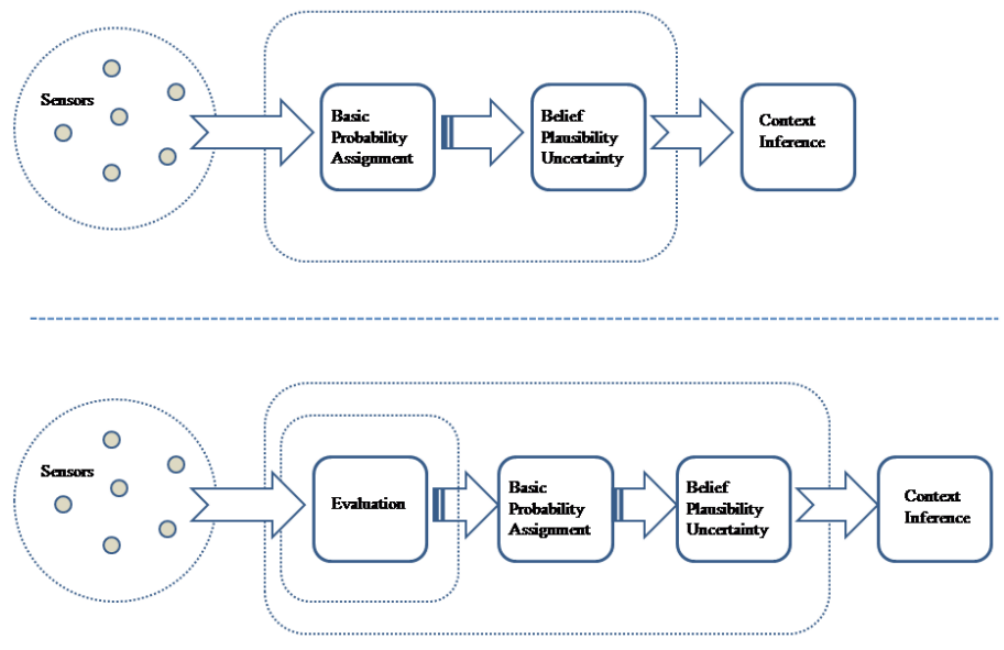

Figure 2. Evaluating signals from sensors 


\subsection{Evaluation criteria for event information}

How to set criteria for evaluating event information is described here.

3.3.1 Event frequency: The first criterion for evaluation is the frequency of sensor signal events. Each sensor reports events to a host when detected sensing values are worth to report. However, the event information is not likely to be reliable with a few frequencies of the events from many sensors composed sensor network. For example, the reliability level of the event information is poor in the forest fire detection system with various sensors spread through the wide range of the forest if some sensors report event occurrences and some do not. On the other hand, the reliability goes up if most of sensors report events with the equivalent frequencies in a certain area. Therefore, it is reasonable to take the frequency of events from each sensor as a criterion.

3.3.2 Variation rates: The differences and directions among data changes of event occurrences reported by sensors in each time interval are the factors to focus on. It is an important clue for context inference to know if the events are increasing or decreasing in time intervals and how big is the differences when they increase. It is better to observe change patterns in the next time intervals when context inference is vague because the differences and directions (positive or negative) of the changes are irregular or uncertain. So, it is necessary to evaluate event information based on event information data variation rates in time intervals of event information reported by sensors.

\subsection{Evaluation procedure of event information}

The procedure to evaluate event information is conducted by the time and criteria described earlier as the following.

1) Detecting event occurrence (Sensor mote)

2) Filtering event information (Sensor mote)

3) Transmitting event information (Sensor mote)

4) Eliminating overlapped event information (Network: Data aggregation)

5) Evaluating event information (Sink nodes: Prior to data fusion)

6) Waiting and saving if event information is inappropriate for fusion process as a result of evaluation

7) Processing data fusion if event information is appropriate for fusion process

8) Inferring contexts after data fusion process

The procedure to evaluate sensing data is presented in Figure 3. 


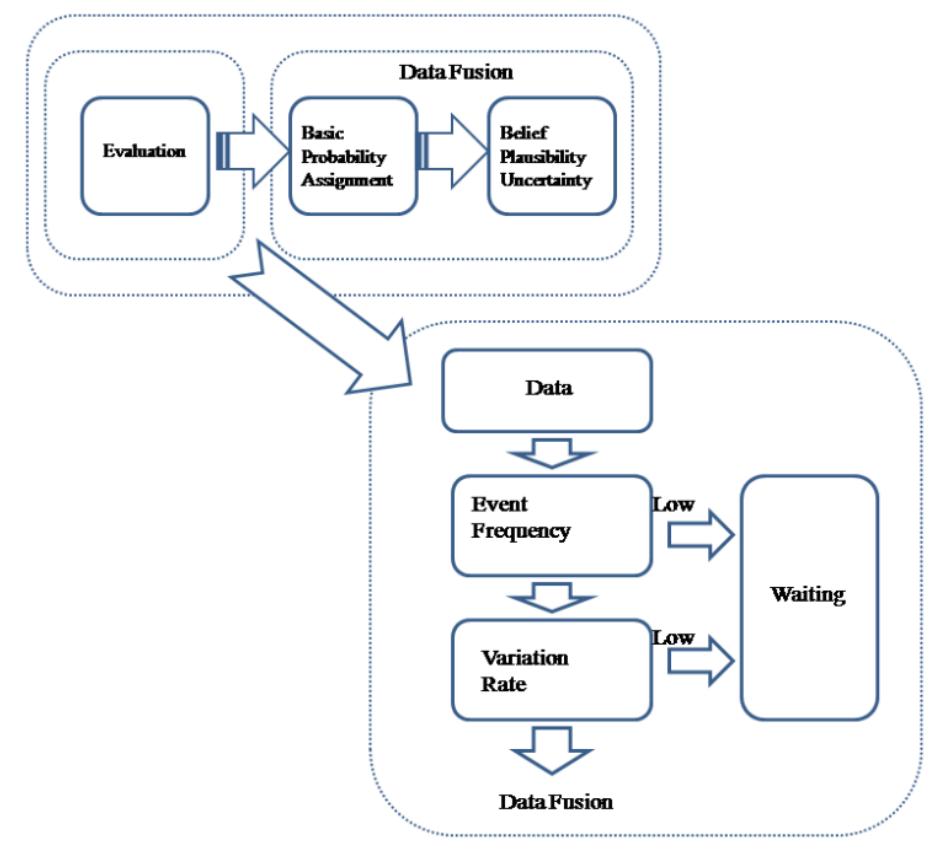

Figure 3. Evaluating procedure of reported data.

\section{Experiment procedure}

\section{Experiment Procedure}

Collect measured values acquired from diverse sensors.

Middleware of sink nodes takes in charge.

Evaluate measured values.

Evaluating criteria is $30 \%$ or more of event occurrence rates out of total sensors, and $0.5 \%$ per a set time of change rates. Multi-sensor data fusion is conducted for the data that passed the evaluation criteria. The data collected in the next intervals with those that did not pass the evaluation criteria are reevaluated. Context inference results are drawn from the fusion process of 5 times or more. The results from the experiment show the following.

Table 1. Fusion results in each interval

\begin{tabular}{lllll}
\hline Focal element & $\mathrm{m}\left(\mathrm{T}^{\prime} 0\right)$ & $\mathrm{m}\left(\mathrm{T}^{\prime} 1\right)$ & $\mathrm{m}\left(\mathrm{T}^{\prime} 2\right)$ & $\mathrm{m}\left(\mathrm{T}^{\prime} 3\right)$ \\
\hline$\Omega$ & 0.30 & 0.30 & 0.09 & 0.00 \\
$\mathrm{~h} 1 \cup \mathrm{h} 2$ & 0.05 & 0.05 & 0.03 & 0.08 \\
$\mathrm{~h} 1 \cup \mathrm{h} 3$ & 0.20 & 0.10 & 0.12 & 0.13 \\
$\mathrm{~h} 2 \cup \mathrm{h} 3$ & 0.25 & 0.25 & 0.22 & 0.18 \\
$\mathrm{~h} 1$ & 0.00 & 0.05 & 0.04 & 0.08 \\
$\mathrm{~h} 2$ & 0.00 & 0.00 & 0.03 & 0.20 \\
$\mathrm{~h} 3$ & 0.20 & 0.25 & 0.46 & 0.33 \\
\hline
\end{tabular}


Table 2. Fusion results in each interval

\begin{tabular}{lllll}
\hline Focal element & bel (T'0) & bel (T'1) & bel (T'2) & bel (T'3) \\
\hline$\Omega$ & 1.00 & 1.00 & 1.00 & 1.00 \\
h1 $\cup$ h2 & 0.05 & 0.10 & 0.10 & 0.36 \\
h1 $\cup \mathrm{h} 3$ & 0.40 & 0.40 & 0.62 & 0.54 \\
h2 $\cup \mathrm{h} 3$ & 0.45 & 0.50 & 0.71 & 0.71 \\
$\mathrm{~h} 1$ & 0.00 & 0.05 & 0.04 & 0.08 \\
$\mathrm{~h} 2$ & 0.00 & 0.00 & 0.03 & 0.20 \\
$\mathrm{~h} 3$ & 0.20 & 0.25 & 0.46 & 0.33 \\
\hline
\end{tabular}

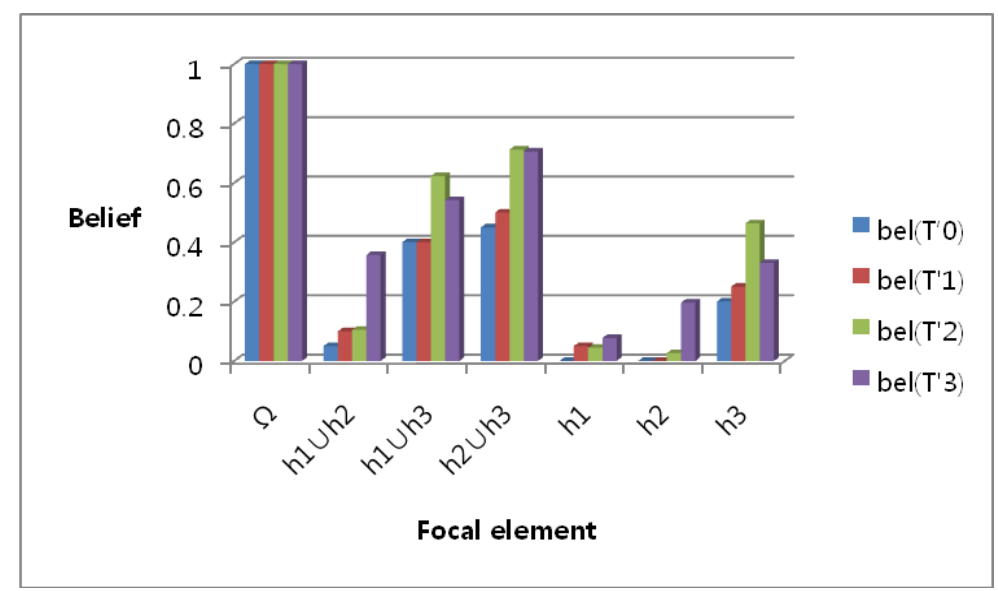

Figure 4. A result of evaluating focal elements

The values detected and reported by temperature sensors were below the evaluation criteria as a result of evaluating before the first time fusion (see Table 1). Multi-sensors data fusion except for temperature values was conducted and the results were reported. Multi-sensors data fusion is processed when the values from all sensors passed the evaluation criteria before the second time fusion, and Basic Probability Assignment (BPA) was calculated in each element. Consequently, the ambiguity of signals reported from sensors was eliminated. Multi-sensors data fusion with only verified sensing values with high reliability achieved the effects of refining context awareness resources.

\section{Conclusion}

Researches on context awareness using signals detected by multi-sensors are undertaken actively in recent years. However, there might be factors lowering the reliability of the signals detected and reported by sensors. The typical examples to damage the reliability of the values detected and reported by sensors are the difficulties and errors occurred in the sensors' detection activities and reporting processes. The result of context awareness would be based on inaccurate data with low level of reliability in these circumstances. Therefore, it is meaningful to evaluate if acquired sensing values are reliable before processing multi-sensors data fusion for context 
awareness. The study presented the evaluation criteria for reliability evaluation of the values detected and reported by sensors before data fusion processes. The reliability is evaluated based on sensors' event occurrence rates and change patterns for certain period of time. The quality of context information was improved for processing data with only sensing values with verified reliability.

\section{References}

[1] T. Gu, H. Pung and D. Zhang, "A Service-Oriented Middleware for Building Context -Aware Services", Jouranal of Network and Computer Applications, vol. 28, no. 1, (2005) January, pp. 1-18.

[2] H. Chen, F. Perich, T. Finin and A. Joshi, "SOUPA: Standard Ontology for Ubiquitous and Pervasive Applications", Proc. of the First Annual International Conference on Mobile and Ubiquitous Systems, Networking and Services, vol. 6, (2004) September, pp. 258-267.

[3] P. Korpipaa, J, Mantjarvi, J. Kela, H. Keranen and E. Malm, "Managing Context Information in Mobile Devices", IEEE Pervasive Computing, vol. 2, no. 3, (2003) July, pp. 42-51.

[4] M. Roman, C. Hess, R. Cerqueira, A. Ranganathan, R. Campbell and K. Nahrstedt, "Gaia: A Middleware Platform for Active Spaces", ACM SIGMOBILE Mobile computing and Communications Review, vol. 6, no. 4 , (2002).

[5] H. Jang and S. Hong, "Study on the OSMU (One-Source Multi-Use) Management for Smart Devices", International Journal of Smart Home, vol. 7, no. 1, (2013) January, pp. 1-8.

[6] Y. -H. Seo, "Face Detector using Network-based Services for a Remote Robot Application", International Journal of Smart Home, vol. 6, no. 2, (2012) April, pp.53-58.

[7] K. -H. Kim, "DSP-based Sequential Parameter Estimation of PWM Inverter-fed IPM Synchronous Machine for Auto-tuning Applications", International Journal of Control and Automation, vol. 6, no. 2, (2013) April, pp. 195-204.

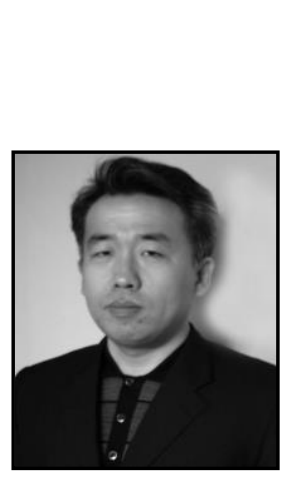

\section{Authors}

\section{Donghyok Suh}

He received the M.S. degrees in computer engineering from Hoseo University in 2005 and the Ph.D. in computer science from Chungbuk National University in 2012. His research interests included in stream data processing and data fusion in wireless sensor network.

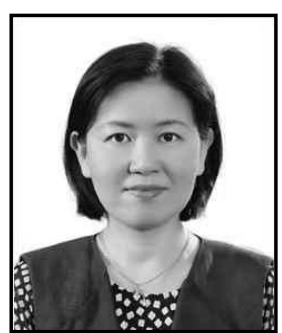

\section{Juhye Yook}

She received the M.Ed. in 1995 and the Ph.D. in 2000 in Special Education from the University of South Carolina in the U.S. She worked for the Korea Employment Promotion Agency for the Disabled (2000 2004) before working at the Korea Nazarene University up until now. Her research interests are computer/information access and assistive technology. 